concentration it will still be useful because it is "exquisitely responsive to minor changes in antigenic determinant structure" and can evidently detect more subtle degrees of damage than can at present be quickly estimated by conventional histological means. Some of these possible secondary effects could be excluded by using it for a time sequence study of spleen in the four weeks after injection, when the concentration of the agent is rapidly increasing there but long before gross signs of damage appear in the brain, or, better, to compare the new and conventional assays using the tissue culture line developed by Haig and Clarke (Nature, 234, 106 ; 1971).

But even in these cases the difficulty remains because of the high sensitivity of their technique, which may be detecting antigens from unseen damage. Its value will only be established when it can be shown that there is a close correlation between the titre estimates of the conventional assay and this new one in a large range of circumstances. This range will need to include various severe physical and chemical treatments of tissue preparations, which could alter the antigenic spectrum without removing all the infectivity. If a close correlation cannot be established between the two types of assay, it will be necessary to await the purification of scrapie as an antigen.

It may therefore be premature to use the method for collateral evidence that there is more scrapie agent in neuronal than glial preparations, as is done in the accompanying communisation by Narang, Shenton, Giorgi and Field (page 106). At this stage standard evidence of infectivity titre is also needed for these two cell populations, and this would give further confidence in the use of the proposed assay. Meanwhile it is difficult to assess the significance of their electron micrographs of particles, but the detection of these in serial passages using only boiled inocula would be more impressive than other evidence.

As the lymphocyte sensitization assay takes only a few days it should be possible to deal quite quickly with some of the points raised here, even though, as Field describes elsewhere, the technique involves "a most fickle and unstable instrument which makes great demands upon the patience and endurance of the observer".-From a Correspondent.
PROTEINS

\section{Further Phosphorylations}

from our Molecular Biology Correspondent

THE role of phosphorylation in the control of glycogen metabolism, by way of cyclic AMP-dependent protein kinase, phosphorylase kinase and glycogen phosphorylases $a$ and $b$, has by now been absorbed into the canon of biochemistry. There is now the glimmer of an intimation that phosphorylation may also take another role in muscle, related in fact to the contractile process.

The discovery that troponin, which is a part of the calcium-driven relaxing system of muscle, is prone to phosphorylation by endogenous enzymes has been reported simultaneously from two laboratories. Pratje and Heilmeyer (FEBS Lett., 27, 89; 1972) have prepared the relaxing protein mixture from myofibrils and observed the incorporation of radioactive phosphate from $\gamma$ labelled ATP when cyclic AMP was present. The relaxing system contains the helical protein tropomyosin and troponin. The latter, as is now known, contains three different chains, of defined function: the inhibitory component (TNI) inhibits actomyosin ATPase; the second, the calcium-binding component (TNC), reverses the inhibition regardless of whether or not calcium is actually bound; and the third (TNT), when combined with the other two, restores the control of the actomyosin ATPase by calcium ions, in that it apparently governs the binding of the inhibitory chains to tropomyosin.

The three proteins differ in molecular weight, and are therefore separable in SDS-acrylamide gel electrophoresis. Phosphorylation proceeds slowly in this system under the stimulus of cyclic AMP, because of the presence of traces of protein kinase. When purified protein kinase is added to the mixture, the reaction goes rapidly, and the location of label in the electrophoresis gels after incubation shows that actin and one of the troponin components only are appreciably phosphorylated. This is the third, and highest molecular weight, chain (TNT). There is at this stage no indication that the phosphorylation changes the relaxing activity of the system in vitro, and the control may be exerted at an earlier stage, in making the phosphorylated component more or less available where it is wanted.

Meanwhile Krebs and his colleagues have looked for, and demonstrated, phosphorylation of troponin under the action of the (in general) much more specific enzyme phosphorylase kinase, the only good substrate for which hitherto known was glycogen phosphorylase b. Stull, Brostrom and Krebs (J. Biol. Chem., 247, 5272 ; 1972) now find that in the presence of ATP and magnesium the enzyme causes phosphorylation - again of only one of the troponin chains, the inhibitory component. Moreover, the enzyme works in both its forms, activated and unactivated (that is to say, whether it is itself phosphorylated by protein kinase

\title{
Gene Duplication in Proteins
}

IN next week's issue of Nature New Biology (November 15), Kretsinger gives evidence of internal duplication of parts of the polypeptide chain of the fish muscle calcium-binding protein, the structure of which was recently solved at high resolution, and McLachlan considers the nature of, and possible tests for, such phenomena. Gene duplication within a single chain has been reported in a number of cases. In the calcium-binding protein, mere inspection of the sequence reveals no obvious homologies. There are two calciumbinding sites, with similar ligands for the ion, but which are not obviously related in sequence. Kretsinger finds, however, that if the three-dimensional structures in these regions are superimposed, the geometrical similarity becomes obvious. Even the backbone carbonyl ligand to the calcium, which is provided in one case by a phenylalanine and in the other by a lysine residue, falls into place. As in other proteins of known structure, such as staphylococcal nuclease and thermolysin, the calcium-binding mode is un- related to that of the muscle protein, the argument against converging evolution is very strong. Thus it seems that structural homologies may transcend divergence of sequences. It also suggests that more versatile methods of searching for sequence homologies are needed, and this is the subject of McLachlan's communication. Using a scoring system for the degree of similarity of the various amino-acids, a homology in the two regions of the calcium-binding protein emerged, and McLachlan has also devised a way of testing for geometrical homologies in terms of the $\alpha$ carbon coordinates along the chain. Kretsinger suggests that a third region in the molecule, which is not implicated in the binding of calcium, is structurally related to the two calcium-binding segments, but McLachlan regards this as less certain. It is suggested that hitherto unsuspected structural homologies, which are only weakly reflected in the sequences (unless a sensitive test is applied), may eventually be shown to be present in many proteins. 